$a, b$. This change corresponds to an invariant subtraction of all diverging terms ; the term $a^{*} k^{n} a_{k^{\prime}} a^{*} k^{\prime} a_{k}$ in $\alpha_{C}{ }^{(*)}$ contributes to the 'Coulomb' self-energy of the $u$-particles. The other terms of $\varepsilon^{n} \alpha^{(n)}$ correspond to the transition-probabilities for collisions in which $n+2$ particles take part (in the sense of chemical reactions). For example, $\varepsilon^{2} \alpha^{\left({ }^{3}\right)} R$ contains the term $a^{*} k^{\prime \prime \prime} a^{*} k^{\prime \prime} a_{k^{\prime}} a_{k}$, where two particles with the momenta $k$ and $k^{\prime}$ disappear and two particles of momenta $k^{\prime \prime}$ and $k^{\prime \prime \prime}$ are created. This is Rutherford scattering (if $\varphi$ is the electromagnetic field) and $\left.\varepsilon^{2} \alpha{ }^{2}\right)_{C}$ contains the Compton effect $\left(a^{*} k^{\prime} b^{*} \mu^{\prime} a_{k} b_{\mu}\right)$, where a $\varphi$-quantum of momentum $\mu$ collides with a $u$-particle of momentum $k$. The terms in $\varepsilon^{3} \alpha^{(3)}$ contain the Bremstrahlung $\left(a^{*} k^{\prime \prime \prime} a^{*} k^{*} b^{*}{ }_{\mu} a_{k^{\prime}} a_{k}\right)$, where, in addition to the Rutherford scattering, a $\mu$-quantum is created.

Putting $t=+T$ and passing to $T \rightarrow \infty$, our theory takes the Heisenberg form. However, we have unambiguously determined the Hermitic Heisenberg operator $\eta=i \varepsilon \alpha(\infty)$ and, furthermore, for any given $t$, we can describe the quantum mechanical state of the system.

We can arbitrarily change the numerical coefficients of each individual $\varepsilon^{n} \alpha^{(n)}$ (or of their invariant parts) without destroying the eonservation laws or the invariance. For example, a theory which contains only $\varepsilon \alpha=\varepsilon^{2} \alpha^{\left({ }^{2}\right)} R$ and nothing else shows Rutherford scattering but no radiation effects (no Compton effect and no Bremstrahlung, etc.). But such a theory is possible even classically. Consider a system of particles, where the force acting upon any one of them is the mean value between the advanced and retarded effect of all other particles. Such a theory is invariant and conserves energy and momentum, but it is not conformal to our causal representation of phenomena. The same acausal behaviour is contained in the quantum mechanical $\Psi(t)$. There exists now a finite probability that a quantum appears at a certain event $(\vec{x}, t)$, without a finite probability that a cause has occurred at a preceding event in the invariant past of $(\vec{x}, t)$. In electrodynamic phenomena, the causal behaviour has been experimentally checked. Therefore, our theory is unambiguous if applied to quantum electrodynamics. Applied to nuclear forces, however, we have great liberty in the choice of $\varepsilon \alpha$ (or of Heisenberg's $\eta$ ) if we go back to a causal description at small distances. Brt one must say that it is not necessary to go back to causal deseription even for distances of $10^{-13} \mathrm{~cm}$.

We have applied our theory to the case of line width. A classical point particle with an internal (scalar) degree of freedom $(\tau, \rho, \sigma)$ treated according to Dirac's method leads to

$$
\ddot{\tau}+\frac{1}{2 \pi} \varepsilon^{2} \mu_{0} \sigma \dot{\tau}+\mu_{0}{ }^{2} \tau=\varepsilon \sigma \varphi(q(\lambda))^{\text {inc } ; \sigma \sim 0 .}
$$

Its line broadening due to radiation damping is (if $\sigma=+1$ and $x=0$ ) therefore

$$
J(\mu)=\gamma^{2}\left(\left(\mu-\mu_{0}\right)^{2}+\gamma^{2}\right)^{-1} ; \gamma=\frac{\varepsilon^{2}}{4 \pi} \mu_{0} \text {. }
$$

The corresponding quantum mechanical model is given by two fields of matter $u$ and $v$ the rest masses of which differ by $m_{v}^{2}-m_{u}^{2} \cong 2 m \mu_{0}$. With $\varepsilon H=2 \varepsilon m \int(d x)^{3} u v \varphi$, only particles of mass $m_{u}\left(<m_{v}\right)$ are stable (analogous to $\sigma=+1$ in classical theory). Exeitation of the $u$-particle into the $v$-state and subsequent emission of a $\varphi \cdot q u a n t u m$, or the dispersion of a $\varphi$-wave produces $\varphi$-quanta of frequency $\mu$ with a probability given by

$$
J(\mu)=\left(\sin \frac{\gamma}{\mu-\mu_{0}}\right)^{2} ; \gamma=\frac{\varepsilon^{2}}{4 \pi} \mu_{0}
$$

instead of (9). This is a rigorous solution of the quantum-mechanical problem. The approximate treatment of Wigner and Weisskopf ${ }^{3}$ leads to (9). Total intensity of the emitted light and the dispersion for $\left|\mu-\mu_{0}\right| \gg \gamma$ are, however, the same.

${ }^{1}$ Dirac, Proc. Roy. Soc., A, 167, 148 (1938).

Heisenberg, Z. Phys., 120, 513 and 673 (1943).

- Weisskopf and Wigner, $Z$. Phus., 63, 54 (1930).

\section{BEHAVIOUR OF THE SONG SPARROW AND OTHER PASSERINES}

$\mathrm{M}$ RS. NICE is well known to ornithologists as an indefatigable and scientific observer, whose previous detailed studies on the song sparrow (Melospiza melodia), published in 1937 as vol. 4 of the Transactions of the Linncean Society of New York, had shed new light on the detailed behaviour of particular individuals of a particular bird species in Nature, as well as extending our general ideas on the territory theory. In a second work* she brings together under their various heads the behaviour traits of the song-sparrows she has watched in Nature and those she has hand-reared herself (all her observations concern colour-banded and therefore individually recognizable individuals), together with a vast amount of data on other species from the recent literature. Thus we have here one of the first essays in the comparative study of avian behaviour.

The result is of great value. Methodologically it is of interest as a crowning demonstration of the fact that field observation, if properly carried out, is an essential tool of biological science: laboratory research could never have elicited the facts and principles here set before us. It is also a reminder for the average biologist that the principles of vertebrate behaviour are now emerging with some clarity, thanks very largely to the labours of field ornithologists, and that they are in many ways unexpected and of great general interest.

It is impossible in the space available to give a critical review, and I must confine myself to citing some of the chief topies covered by Mrs. Nice, with the reminder that in each case she gives a balanced diseussion of her own and others' findings.

These topics include an attempt to disentangle innate and acquired factors in bird behaviour (which leads to somewhat surprising results); a general discussion of Lorenz's basic theories of releaser or signal stimuli, and of the role of the Kumpan (or fellow-member of the same species) in the social life of birds ; an account of the genetic psychology of passerine birds from hatching onwards; waking and sleeping times in relation to twilight; a discussion of the dominance and subordination relations of passerines; 'symbolic' actions ; song; territory ; habitat selection; a general analysis of the difficult problems of pair-formation; courtship ; injuryfeigning ; enemy recognition ; individual variation in behaviour.

* "Studies in the Life History of the Song sparrow (2). The Behavior of the Song Sparrow and other Passennes". By Mrs. M. M. Nice. (Trans. Linn. Soc., N.Y., 6, Sept. 1943, np. viit +329.) 
In regard to this last point, Mrs. Nice makes it clear that every individual male song-sparrow has his own repertoire of songs, all of them readily distinguishable, with a little practice, from those of all other males. The number of separate songs in a cock bird's repertoire varies from six to twentyfour. One male song-sparrow sang from 4.45 a.m. to 7.43 p.m., giving 2,305 songs in fifteen hours, with 278 songs as his highest rate per hour !

Again (as Lack has since shown in the English robin) migration is a matter of individual variation; and indeed birds that migrate one year need not do so the next.

There are a few criticisms to be made. Among several misprints (including some, such as the transposition of lines on p. 64, which one does not expect to find in a scientific publication), there is one serious one in Table 13 where songs should apparently be song-series ; and in Table 5, the "group V" discussed in the text is omitted. Although a great volume of literature is comparatively reviewed, there are some curious and unexplained gaps. Thus, though Lloyd Morgan's fundamentel "Habit and Instinct" and Kirkman's recent important studies on egg and nestsite recognition are cited in the literature list, they appear to be neglected in the discussion. The important work of Lockley on homing and other activities in shearwaters is entirely omitted, together with the numerous data of various authors on the behaviour of the gannet, and the valuable work of Nicholson and Koch, complete with gramophone records, on the song of British birds. More generally, the sections on courtship and display might have been fuller, with more weight given to studies on other forms, notably non-passerines.

These, however, are mere minor omissions. The work as a whole is a monument of industry and a model of method. If the author appears to go a little far in claiming (p. 273) that "the study of animal behavior is the only and ultimate souree of understanding ourselves", it is certainly true that it is an indispensable aid in that task, and further that it reveals unexpected aspects of mental evolution which even the most complete study of our own psychological organization could not have made available. In any event, in this and tha preceding work, Mrs. Nice has made a massive and outstanding contribution to our advaneing knowledge of animal behaviour. Julian Huxidey.

\section{OX BLOOD FOR BLOOD TRANSFUSION}

$\mathrm{M}$ ANY people must have wondered whether some use could not be made of the large quantities of ox blood available in our slaughter-houses. If the conclusions drawn by Dr. Edwards (Brit. Med. $J$. Jan. 15, 1944) and his collaborators survive the further tests to which they are being subjected, the ax of the future may not only give his life and meat for man, but may serve him also with his very blood and may be kept for that parpose by the com. munity.

The use of plasma for surgical treatment is, as Dr. Edwards points out, inereasing rapidly, and the search for a suitable plasma other than that provided by the blood banks is in progress. It would seem that the collaboration between Dr. Edwards and the Regional Transfusion Laboratories, the North Staf- fordshire Royal Infirmary and experts at Cambridge and Liverpool, has gone a long way towards the solution of this problem. The substitutes for human plasma which have been tried have not, Dr. Edwards states, possessed the three characteristics that are necessary, namely, that they should be retained in the circulation and should eventually be metabolized, that they should exert an osmotic pressure equivalent to that of the plasma and that they should be nontoxic, free from antibodies and non-antigenic. Bayliss in 1916 tried 6 per cent gum acacia in saline for the treatment of shock, but this was only partially metabolized and much of it remained in the tissues with unpleasant consequences. Other substances mentioned by Dr. Edwards may maintain the blood. pressure in shock and hæmorrhage, but they do not restore the blood protein. Animal plasma protein most nearly resembles human plasma protein, and the total protein of bovine blood most nearly approaches that of human blood; but it contains a much higher percentage of fibrinogen. Further, its albumin/globulin ratio is lower, so that bovine blood might exert a lower osmotic pressure, because the albumin fraction exerts the greater pressure. Bovine blood is, however, available in practically unlimited quantities.

It has been shown that crude bovine serum is unsuitable. Dr. Edwards outlines the attempts to overcome such difficulties as the serum sickness which it causes and the tendency to hæmolysis of the human red cells and the slow rate of administration. $\mathrm{He}$ explains in detail the method which he and his collaborators have finally adopted for the preparation of a bovine serum which has been tested on twenty-six cases. The method includes the heating of the serum to $72^{\circ} \mathrm{C}$. to destroy the antibodies and the addition of 0.2 per cent of formalin and ammonia to render the proteins uncoagulable. He claims that the serum obtained fulfils the requirernents indicated above.

The final product is called D.B.S. (despeciated bovine serum). It has an osmotic pressure comparable to that of filtered human plasma. It was kept for six months in Dr. Edwards's car and was then given to a patient without untoward results; after nine months in a refrigerator it had not deteriorated. It can, moreover, be given very rapidly and in large amounts. After a preliminary trial on twenty-six patients, Dr. Edwards clairns that it is safe to give it to man and that it is well retained in the circulation. It is easily prepared in large quantities.

The possibility of the transmission by it of tuber culosis or of Brucella abortus has been considered. Dr. Edwards advocates bleeding from tubereulintested stock only; or only from bullocks and heifers, because only 6 per cent of these coming to the slaughter-house are infected with tuberculosis or Brucella abortus, whereas the rate among slaughtered cows is 30 per cent. If the meat inspector reports gross tubereulous infection of the carcass, the blood taken from that animal should be discarded. But the Seitz filtration during the process of manufacture should, Dr. Edwards thinks, remove any of these organisms that may be present in the blood.

Before he gives a final judgment, Dr. Edwards wisely awaits the results of more detailed hrematological and biochemical work on the effects of the administration of despeciated blood serum to patients in states of shock, hypoproteinæmia and protein deprivation and loss. G. LAPAGE. 